\title{
Projeto de ensino como apoio ao telemonitoramento dos casos de Covid-19
}

\author{
Teaching project to support telemonitoring of Covid-19 cases
}

\author{
Rodrigo Pinheiro Silveira' (D) ropsilveira@gmail.com \\ Joicely Melo da Costa' (D) joicelycosta@uol.com.br \\ Siglia Sousa de França' (D) sigliafranca@gmail.com \\ Rita de Cássia Ribeiro Pereira' ${ }^{1}$ (D) ritapereira_17@hotmail.com \\ Leonardo Assad Lomonaco' (1) leonardoalomonaco@yahoo.com.br \\ Osvaldo de Sousa Leal Junior ${ }^{1}$ (D) $\mid$ ms.osvaldo@uol.com.br
}

\section{RESUMO}

Introdução: Em janeiro de 2020, a OMS reconheceu a pandemia do novo coronavírus no mundo, chegando os casos ao Brasil em fevereiro e ao Acre em março. Uma das respostas para enfrentamento da pandemia no estado foi o telemonitoramento dos casos suspeitos e confirmados de Covid-19, que se viabilizou a partir da parceria entre o Núcleo Telessaúde Acre, as Secretarias de Saúde e os cursos Medicina da Universidade Federal do Acre (Ufac) e do Centro Universitário Uninorte. O objetivo do artigo é relatar a experiência do projeto de ensino de apoio ao telemonitoramento dos casos de covid-19 em Rio Branco.

Relato de experiência: $O$ telemonitoramento tem sido realizado por uma equipe composta de 210 alunos de Medicina dos últimos períodos do curso e por um grupo de oito professores. Atenta às necessidades de fortalecimento das ações de enfrentamento do novo coronavírus, a PróReitoria de Graduação da Ufac lançou um edital para projetos de ensino que pudessem cumprir esse papel social por meio da sistematização de conhecimentos sobre o tema.

Discussão: A partir daí, o projeto foi executado através de reuniões virtuais sistemáticas da equipe executora do telemonitoramento pela plataforma Zoom, com discussões dos casos acompanhados, de rodas de conversa com especialistas sobre temas clínicos específicos, apresentação de artigos, discussão de dados epidemiológicos e aulas expositivas sobre a Covid-19. Além disso, têm sido articulados trabalhos de conclusão de curso a partir dos dados trabalhados na estratégia.

Conclusão: Apesar do desafio que é trabalhar a partir do ensino remoto, o projeto tem contribuído de maneira substancial para o aprofundamento dos conhecimentos sobre a pandemia de Covid-19 e para o acompanhamento dos casos em Rio Branco.

Palavras-chave: Educação Médica; Telemonitoramento; Infecções por Coronavírus.

\section{ABSTRACT}

Introduction: In January 2020, the WHO recognized the new Coronavirus pandemic, reaching Brazil in February, and the state of Acre in March. One of the actions employed to tackle the pandemic in Acre is the telemonitoring of suspected and confirmed Covid-19 cases, enabled though a partnership between the Acre Telehealth Center, the Health Departments and the two local medical courses. The purpose of this article is to report the experience of the teaching project to support telemonitoring of Covid-19 cases in Rio Branco.

Experience report: The telemonitoring was performed by a team of 210 final year medical student and a group of eight professors. Aware of the need to strengthen actions to tackle the new Coronavirus, the UFAC central administration issued a public notice for teaching projects that could fulfill this social role by systematizing knowledge on the subject.

Discussion: The project has been carried out through systematic virtual meetings of the telemonitoring executive team on the Zoom platform, with discussions of the monitored cases, discussions with specialists on specific clinical topics, the presentation of articles, discussion of epidemiological data and lectures on Covid-19. Furthermore, course conclusion papers have been developed based on the telemonitoring data.

Conclusion: Despite the challenge of working through distance learning, the project has contributed substantially to deepening knowledge about the Covid-19 pandemic and to monitoring cases in Rio Branco.

Keywords: Education Medical; Telemonitoring: Coronavirus Infections.

\footnotetext{
${ }^{1}$ Universidade Federal do Acre, Rio Branco, Acre, Brasil.
}

Editora-chefe: Daniela Chiesa

Editora associada: Rosiane Viana Zuza Diniz

Recebido em 14/08/20; Aceito em 29/01/21.

Avaliado pelo processo de double blind review. 


\section{INTRODUÇÃO}

Em 30 de janeiro de 2020, a Organização Mundial da Saúde declarou estado de emergência em saúde pública pelo novo coronavírus (severe acute respiratory syndrome coronavirus - Sars-CoV-2), após reconhecimento da pandemia da coronavírus disease 2019 (Covid-19), causando grande impacto sanitário, econômico e social em praticamente todos os países do mundo'. Os casos de coronavírus chegaram ao Brasil oficialmente em fevereiro de 2020, e, no dia 14 de março, foi notificado o primeiro caso no estado do Acre. Desde então, o número de casos novos cresceu exponencialmente e se estabilizou em um grande platô. O boletim do dia 6 de agosto registrou 21.263 casos confirmados no Acre, sendo 9.393 em Rio Branco.

A partir do início do isolamento social ocorrido no mês de março, houve paralisação das atividades comerciais e educativas, incluindo as aulas e o internato dos cursos de Medicina do estado, da Universidade Federal do Acre (Ufac) e do Centro Universitário Uninorte. Aproveitando o momento, professores e alunos se articularam em parceria com o Núcleo Telessaúde Acre para a realização do telemonitoramento dos casos. Após seu início, essas ações se configuraram como projetos de extensão da Ufac, proporcionando o pagamento de bolsas para os alunos envolvidos.

O telemonitoramento é o acompanhamento dos casos suspeitos e confirmados de Covid-19 de Rio Branco, realizado por uma equipe de 210 alunos dos dois cursos, supervisionada por professores médicos das instituições. É uma ação realizada em parceria entre as instituições de ensino e as Secretarias Estadual e Municipal de Saúde de Rio Branco. O início foi no dia 28 de março, quando o Estado tinha assinalado 23 casos confirmados, e o término em 30 de setembro, quando houve queda no número de casos e o envolvimento dos profissionais da atenção primária na ação.

Atenta às ações de extensão desenvolvidas e empenhada no sentido de fortalecê-las, a Pró-Reitoria de Graduação da Ufac lançou um edital para a seleção de projetos de ensino que pudessem apoiar as ações desenvolvidas no enfrentamento à pandemia no estado. Naquele momento, já se sentia a necessidade de aporte didático-científico à estratégia, que pudesse ampliar os conhecimentos sobre o coronavírus e os aspectos fisiopatológicos e clínicos da Covid-19. Aproveitando essa oportunidade o grupo de professores e alunos se articulou novamente e submeteu o projeto "Telemonitoramento Covid-19 - ações de ensino-aprendizagem para internos de Medicina", que foi aprovado, e possibilitou a aquisição de mais 40 bolsas por três meses de trabalho.

O objetivo do presente artigo é relatar a experiência do projeto de ensino de apoio ao telemonitoramento dos casos de Covid-19, ressaltando a oportunidade de integração entre ensino, pesquisa e extensão, além da utilização de novas possibilidades de ensino-aprendizagem em ambiente virtual.

\section{RELATO DE EXPERIÊNCIA}

Com o aumento do número de casos em Rio Branco, houve estruturação do fluxo de atendimento, tendo as ações de telemedicina papel central na assistência e vigilância dos casos, em um momento em que toda a rede de atenção primária se envolveu no enfrentamento da pandemia.

Os objetivos do telemonitoramento se concentraram nos aspectos clínicos e epidemiológicos dos casos. Depois da disseminação do vírus em fase de transmissão comunitária, houve maior enfoque na evolução clínica das pessoas acometidas pelo vírus. Sendo assim, além das orientações de isolamento e detecção de contactantes, a ação teve seu cerne na avaliação de risco e gravidade dos casos, identificando aqueles que podiam permanecer em acompanhamento domiciliar, os que deviam ser encaminhados para avaliação presencial, e aqueles que precisaram de atendimento de urgência e emergência. Dessa maneira, atuou na coordenação do cuidado dos casos confirmados e suspeitos de Covid-19 ao longo do processo de adoecimento, possibilitando o acesso oportuno aos recursos disponíveis no sistema de saúde.

Os casos foram captados por meio das planilhas de casos confirmados enviados pela coordenação de vigilância do município, da identificação contactantes sintomáticos ou repassados de outras unidades. Os casos eram então enviados aos alunos para se iniciar o acompanhamento.

Para realizar o telemonitoramento, os alunos de Medicina utilizaram o seu próprio número de telefone/WhatsApp, entrando em contato por mensagem de texto ou ligação. A partir do diálogo, era preenchido um formulário Google Forms construído especificamente para a estratégia, com informações pertinentes ao caso. Com esses dados, foi possível classificar os pacientes em relação à gravidade e ao risco, determinando a conduta a ser seguida. Uma descrição detalhada dessa ação pode ser encontrada em Silveira et al. ${ }^{2}$.

A ação de telemonitoramento durou seis meses. No total, selecionaram-se para acompanhamento 8.298 casos, dos quais 7.816 foram confirmados, representando $80,5 \%$ dos casos do município (não se incluíram os casos confirmados por teste rápido). Do total, houve 32\% de perdas (não responderam, recusas e contatos errados), e 3.847 casos tiveram alta do monitoramento.

Desde o primeiro mês de trabalho, já se detectou a necessidade de reuniões periódicas com a equipe no sentido de qualificar a ação e aprofundar os conhecimentos sobre a Covid-19. Os encontros começaram antes mesmo do resultado do edital de projetos de ensino, com o propósito de obter o apoio 
necessário para a consolidação e o fortalecimento da equipe. O grupo de professores foi bastante diversificado, havendo especialistas nas áreas de medicina de família e comunidade, cardiologia, infectologia, hematologia, gastroenterologia, ginecologia e obstetrícia, pediatria e radiologia. Essa diversidade de áreas médicas proporcionou uma ampliação do olhar, o que permitiu a exploração de diferentes pontos de vista sobre o mesmo fenômeno.

O projeto de ensino foi composto de cinco diferentes ações, sendo a principal a sessão clínica do telemonitoramento, fruto dessas reuniões iniciais realizadas. Além dessa ação, o projeto abrangeu também os seguintes movimentos: atividade de orientação e preceptoria, que consistiu nos contatos cotidianos entre alunos e professores sobre os casos acompanhados, principalmente por via aplicativo WhatsApp; a sistematização do conteúdo do registros de acompanhamento, o que possibilitou a produção de relatórios e trabalhos científicos a partir dos dados produzidos no monitoramento; a participação em webpalestras sobre coronavírus organizadas pelo Núcleo Telessaúde Acre; e a ação de proteção dos grupos de risco, realizada com a produção de vídeos informativos, veiculados pelos alunos para os pacientes ao longo do processo de monitoramento. Neste artigo, optamos por abordar a ação principal pelo seu caráter estruturante dentro do projeto.

As sessões clínicas foram realizadas desde o dia 16 de maio, de maneira sistemática, três vezes por semana, às terças e quintasfeiras à noite e aos sábados à tarde, até o dia $1^{\circ}$ de outubro, quando ocorreu a última; nesse momento final, avaliou-se toda a ação do telemonitoramento e houve debate sobre as perspectivas futuras da pandemia. No total, realizaram-se 45 encontros, perfazendo 90 horas de carga horária. Os encontros ocorreram sempre em ambiente virtual, por meio da plataforma Zoom. Os professores e alunos se reuniram principalmente em torno dos casos acompanhados no telemonitoramento, mas houve utilização de outras metodologias, como "rodas de conversa", apresentação de artigos, discussão sobre indicadores epidemiológicos, gestão do sistema de saúde, além de aulas expositivas.

Como marco inicial da sessão, foi apresentado e debatido no grupo o protocolo de manejo da Covid-19 do Acre $^{3}$, o que possibilitou uma aproximação com o conjunto de evidências sobre fisiopatologia, quadro clínico (com destaque para as fases da doença), avaliação laboratorial, diagnóstico e tratamento. Houve a exposição por infectologista, e o debate aconteceu com foco na realização do telemonitoramento. Dessa maneira, a ação foi totalmente redirecionada em conformidade com as recomendações do documento.

Na discussão, a proposta foi fazer uma grande "visita" virtual aos casos acompanhados. Os alunos eram convidados a apresentar os casos, com destaque para aqueles em que havia maior risco, gravidade, complicações ou manifestações clínicas atípicas da Covid-19. Dessa forma, pôde-se explorar ao máximo o aprendizado a partir da prática do acompanhamento dos casos. Para apresentação, foi feita orientação sobre os dados mais importantes, para que houvesse clareza na compreensão dos participantes. A descrição começava com as letras iniciais do paciente, a idade, a menção sobre a existência ou não de comorbidade e o tempo de doença. Na sequência, fazia-se um resumo sobre a evolução do quadro clínico (sinais e sintomas), as medicações em uso, a evolução e a situação atual, como mostra o exemplo da Figura 1. O mesmo exemplo foi utilizado para encaminhamento do paciente para unidades de referência. Esse modelo de descrição se tornou a marca da sessão clínica.

Figura 1. Exemplo de resumo do caso para discussão

\section{TELEMONITORAMENTO COVID-19}

Homem, 55 anos, hipertenso, 11 dia de doença, teve febre tosse, coriza e odinofagia nos primeiros dias. Utilizou Azitromicina, Hidroxicloroquina e Oseltamivir por 5 dias. Evoluiu melhora desses sintomas permanecendo apenas com tosse seca. Há 2 dias houve piora clínica, com dor torácica e dispneia aos médios esforços. Procurou unidade de referência para Covid. Diz que a SatO2 estava $97 \%$, sendo prescrito Enoxaparina $40 \mathrm{mg} / \mathrm{dia}$ e solicitados exames. Fez TC que revelou opacidades em vidro fosco acometendo $25 \%$ do pulmão. Exames mostram linfopenia discreta, PCR 30, LDH e Transaminases normais, D-dímero levemente alterado. No momento ainda com dispneia leve, FR $24 \mathrm{inc} / \mathrm{min}$.

Fonte: Elaborada pela equipe de telemonitoramento do Núcleo Telessaúde Acre. 
Após a apresentação, havia comentários dos professores e alunos, tanto por fala, como pelo fórum, que foi também utilizado para a postagem de casos para que todos acompanhassem a leitura. Ao final da discussão, os alunos eram orientados sobre a conduta em relação ao caso, quando apresentavam as possibilidades de encaminhamento, se fosse o caso, e já faziam o contato com o local de referência. Embora tenha sido realizada em ambiente virtual, havia preocupação sobre os cuidados com a segurança dos dados e as questões éticas envolvidas para que não houvesse exposição de dados que identificassem os pacientes.

Das atividades realizadas, as mais interessantes foram as rodas de conversa com especialistas ou profissionais envolvidos no enfrentamento da Covid-19, de diferentes áreas e inserções dentro do sistema. Essas atividades começavam como uma entrevista, mas com espaço para conversas informais, para falas dos participantes e perguntas no fórum. Nesse formato, foram realizados encontros com professores das áreas de farmacologia, clínica médica, cardiologia, infectologia, medicina de família e comunidade, fisioterapia, entre outras. Destaca-se aqui uma sessão realizada com um médico que foi infectado pela Covid-19 e que necessitou de internação hospitalar. Nessa oportunidade, puderam-se discutir aspectos da abordagem centrada na pessoa, como a experiência de adoecimento, além de se tentar entender as particularidades de quando o médico vira paciente.

$\mathrm{Na}$ apresentação de artigos, discutiram-se estudos originais e revisões que abordavam a imunologia da Covid-19, os testes clínicos utilizados para diagnóstico, os sintomas mais frequentes, as opções terapêuticas, entre outros assuntos. Nesse momento, houve aprofundamento do conhecimento e apropriação das evidências mais recentes sobre a Covid-19, especialmente em seu aspecto clínico, o que ajudou bastante na condução dos casos. As apresentações se iniciaram com os professores, mas depois foram ampliadas para os alunos bolsistas do projeto, que, em grupos, apresentavam os artigos para posterior debate. Destaca-se nesse processo o artigo de Carfi et al. ${ }^{4}$, que trata dos sintomas tardios da doença, tópico que suscitou muitas dúvidas.

Apesar de não ser a intenção inicial, o grupo optou por incluir algumas aulas expositivas dentro das sessões. Houve apresentação sobre radiologia da Covid-19, manifestações gastroenterológicas e neurológicas da doença, o que ampliou a compreensão das manifestações extra-pulmonares frequentemente observadas nos casos. Essas aulas trouxeram compreensão de gravidade e manejo dos sintomas, influenciando diretamente nas possibilidades terapêuticas mais direcionadas.

Outros assuntos debatidos na experiência foram os indicadores epidemiológicos e aspectos da gestão em saúde no enfrentamento da pandemia. Com essa discussão, o grupo foi capaz de entender a situação da evolução de casos e óbitos, incluindo as estimativas de subnotificação, os indicadores de testagem, e os índices de transmissibilidade e de isolamento social. Para essas discussões, foram convidados gestores de Rio Branco, fato que possibilitou a participação ativa do grupo na construção e análise do primeiro inquérito soroepidemiológico do município ${ }^{5}$. No grupo discutiu-se também o plano de reabertura do estado ${ }^{6}$, que balizou os processos de flexibilização das instituições em relação ao retorno do funcionamento.

De modo geral, a participação dos alunos no telemonitoramento e no projeto de ensino foi pertinente tanto pela capacidade técnica como pela vivência em aplicativos virtuais de relacionamento. $\mathrm{Na}$ impossibilidade de atuação presencial nos campos de prática, a realização do telemonitoramento possibilitou aos alunos a vivência de ações de cuidado congruentes com o projeto pedagógico do curso, constituindo uma possibilidade de ensino-aprendizagem prática, mas em ambientes virtuais.

A utilização da telemedicina para aproximar o estudante da comunidade já era utilizada anteriormente à pandemia ${ }^{7}$, mas em nossa experiência, considerando a possibilidade de contaminação, o trabalho virtual permitiu que alunos $e$ docentes exercessem as atividades sem se exporem ao risco de contágio pela doença².

O uso do WhatsApp permitiu o gerenciamento do grupo e a troca de informações rápidas entre alunos e professores. Silva et al. ${ }^{8}$ relataram que a maioria dos alunos identificaram maior habilidade para o uso de ferramentas para interação online depois que participaram de um projeto de educação com o uso dessas tecnologias. Em nossa experiência, tanto os alunos quanto os professores já conheciam as plataformas utilizadas, mas a maioria dos docentes tem se aprimorado em sua atuação e em dispositivos específicos que poderão ajudar na adaptação às aulas remotas.

O contexto da pandemia tem desafiado os docentes a reinventar a maneira de ensinar e exercer a medicina. Graças às novas tecnologias digitais, é possível colocar em prática modalidades de ensino de forma remota. Embora seja um desafio para os atores envolvidos, é possível a diversificação de metodologias, colocando os alunos também como protagonistas do processo ensino-aprendizagem, trabalhando com eles as ferramentas para a aquisição do conhecimento e desenvolvimento de habilidades importantes para a formação médica adequada, mesmo no contexto do que consideramos como o "novo normal".

Segundo Ruiz et al. ${ }^{9}$, o e-learning é capaz ajudar a promover a auto-aprendizagem, e ser tão bem-sucedido quanto a didática tradicional, já que é considerado agradável 
por uma parcela expressiva de estudantes de Medicina. Nessa experiência, realizaram-se verdadeiros rounds com a diversificação de métodos capazes de formar um mosaico de conteúdos importantes para o manejo de casos de Covid-19. Dessa forma, o estudante é estimulado a fazer parte da tomada de decisões, realizar aconselhamento de pacientes e familiares, e participar do planejamento de intervenções ${ }^{10}$.

Nesse momento de reorganização dos processos de ensino-aprendizagem e incorporação de métodos de ensino remoto, é importante elencar competências que podem ser adquiridas nesses processos. No trabalho de Silva et al. ${ }^{8}$, $81,9 \%$ dos graduandos de Medicina que participaram de um projeto que utilizou a telemedicina perceberam aumento das suas habilidades de comunicação. Destaca-se que, para esse tipo de atendimento, deve-se fazer compreender por meio da linguagem e ao mesmo tempo ter a capacidade de dialogar sobre a importância das informações científicas de prevenção para conter a disseminação do novo coronavírus. No caso da experiência relatada, essa foi uma das competências mais trabalhadas, inclusive considerando que a comunicação escrita tem as suas particularidades em relação à linguagem oral.

Entre tantas possibilidades o projeto foi fundamental para identificar as necessidades de conhecimento por parte dos alunos, auxiliando em um processo de metacognição, e estimular busca por fontes de atualização. Com as orientações dos docentes, os discentes podem avançar também em análises críticas da literatura, de modo que sejam capazes de transmitir as orientações para os pacientes, e também de participar das reuniões de discussão de casos.

Mesmo com as novas possibilidades, há limites e desafios nessa tentativa de transpor metodologias para o espaço virtual, o que constitui um novo objeto de debates para docentes, discentes e gestores das instituições de ensino. Chinelatto et al. ${ }^{11}$, em um artigo que mostra as perdas e os ganhos no ensino médico durante a pandemia, ressaltam que muito professores ainda têm dificuldades em utilizar as plataformas virtuais e redes sociais, já que tiveram muito pouco tempo para essa adaptação. Além disso, os autores apontam que, principalmente em relação às discussões de casos clínicos, o meio virtual pode diminuir a qualidade do debate. Sem dúvida, outro desafio refere-se à participação discente. No presente projeto, foram feitas reuniões do grupo para correções de rumo e combinação das tarefas, o que resultou em uma melhora da participação e o aumento do protagonismo dos estudantes.

Para além do debate sobre essa nova realidade, mesmo com a distância física, talvez sejam necessárias ferramentas que possam manter a proximidade entre docente e discente, de modo a preservar o clima acadêmico e vincular o afeto ao aprendizado nessa relação. Quando a pandemia findar, é imprescindível a incorporação, de maneira permanente, desses novos caminhos complementares ao encontro presencial, insubstituível na educação médica. O mundo pede dinamismo, e esse talvez seja o gatilho para que mais sessões e discussões virtuais possam encurtar caminhos e melhorar a agilidade sobre condutas e estratégias a serem tomadas na prática clínica.

\section{CONCLUSÕES}

Inicialmente é importante destacar que o lançamento de um edital de projetos de ensino constitui uma inovação no contexto da universidade, havendo influência inequívoca da paralisação e das mudanças no contexto acadêmico decorrentes da pandemia de Covid-19. Os editais com maior visibilidade são de projetos de pesquisa ou extensão. O presente relato mostra que editais de ensino para apoio às ações de extensão representam mais uma possibilidade para a universidade na busca de integração do tripé ensino-pesquisa-extensão.

Para o telemonitoramento, o projeto foi um ponto de apoio que ajudou a sustentar a atuação de um grupo que, em meio ao isolamento social e à paralisação de atividades, empenhou-se em uma ação importante para o enfrentamento da pandemia. Esse apoio contribuiu tanto para a qualificação da estratégia, por meio do aprofundamento dos conhecimentos e da atualização quase em tempo real, como para o aumento da capacidade de resiliência frente ao clima de tensão e medo causado pelo estado de emergência em saúde pública em que a humanidade se encontra. Observou-se então que, além da atuação em domínio cognitivo, a ação contribuiu também para a saúde mental dos estudantes e professores em um momento difícil para todos.

O meio virtual de encontro e de diálogo tem sido o maior desafio para projetos desse tipo. Mas, diante dessa imposição, o projeto contribuiu para o aprimoramento dessa modalidade remota emergencial de ensino, mesmo considerando suas limitações. Essa é uma das mudanças que provavelmente farão parte do legado pós-pandemia para a pedagogia como ciência, tendo como desafio ainda maior o campo da educação médica, em que a prática e a interação são fundamentais.

Por fim, esse movimento proporcionado pelo projeto de ensino em apoio ao telemonitoramento, constitui uma resposta à altura dos desafios que este momento difícil impôs a toda a sociedade e em particular às instituições de ensino superior, sendo capaz de integrar mérito acadêmico e responsabilidade social.

\section{CONTRIBUIÇÃO DOS AUTORES}

Rodrigo Pinheiro Silveira: participou da redação e revisão de todas as partes do texto. Joicely Melo da Costa: participou da redação do relato de experiência e da revisão do texto. Siglia Sousa de França: participou da discussão e da redação do relato 
de experiência. Rita de Cássia Ribeiro Pereira e Leonardo Assad Lomonaco: participaram da redação do relato de experiência. Osvaldo de Sousa Leal Junior: elaborou a introdução do relato de experiência e participou da revisão do texto.

\section{CONFLITO DE INTERESSES}

Declaramos não haver conflito de interesses neste estudo.

\section{FINANCIAMENTO}

Declaramos que não houve financiamento neste estudo.

\section{REFERÊNCIAS}

1. Organização Pan-americana de Saúde. Folha informativa - COVID-19 [acessoem 30maio2020].Disponívelem: https://www.paho.org/bra/index. php?option=com_content\&view=article\&id=6101:covid19\&ltemid=875.

2. Silveira RP, Leal O, Soares PLS, Cruz LF, Modesto IDM, Batista LMB, et al. Telemonitoramento da Covid-19 com participação de estudantes de medicina: experiência na coordenação do cuidado em Rio Branco, Acre. APS em Revista. 2020;2(2):151-61.

3. Acre. Recomendações para o manejo de pacientes no contexto da Covid-19. Rio Branco: Secretaria de Estado de Saúde; 2020.
4. Carfi A, Bernabei R, Landi F. Persistent symptoms in patients after acute Covid-19. JAMA. 2020;324(6):603-5.

5. Rio Branco. Inquérito soroepidemiológico de Rio Branco: relatório final. Rio Branco: Secretaria Municipal de Saúde; 2020.

6. Acre. Decreto $n^{\circ} 6.2026$, de 22 de junho de 2020. Dispõe sobre a criação do Pacto Acre Sem COVID e prorroga prazos previstos no Decreto n 5.496 , de 20 de março de 2020, que estabelece medidas para enfrentamento da emergência de saúde pública decorrente da doença COVID-19. Diário Oficial do Estado do Acre; 22 jun 2020.

7. Machado FSN, Carvalho MAP, Mataresi A, Mendonça ET, Cardoso LM, Yogi MS, et al. Utilização da telemedicina como estratégia de promoção de saúde em comunidades ribeirinhas da Amazônia: experiência de trabalho interdisciplinar integrando as diretrizes do SUS. Cienc Saude Colet 2010;15(1):247-54.

8. Silva DR, Lima LRA, Cara LM, Wen CL. Projeto Jovem Doutor: o aprendizado prático de estudantes de medicina por meio de atividade socioeducativa. Rev Med (São Paulo). 2017;96(2): 73-80.

9. Ruiz JG, Mintzer MJ, Leipzig RM. The impact of e-learning in medical education. Acad Med. 2006;81(3):207-12.

10. Theoret $C$, Ming X. Our education, our concerns: the impact on medical student education of Covid-19. Med Educ. 2020;54(7):591-2.

11. Chinelatto LA, Costa TRD, Medeiros VMB, Boog GHP, Hojaij FC, Tempski PZ, et al. What you gain and what you lose in Covid-19: perception of medical students on their education. Clinics. 2020;75:e2133 